\title{
IMPROVED MPC DESIGN BASED ON SATURATING CONTROL LAWS
}

\author{
D. Limon*, J.M. Gomes da Silva Jr. ${ }^{\dagger}$, T. Alamo* and E.F. Camacho* \\ * Dpto. de Ingenieria de Sistemas y Automática. Universidad de Sevilla \\ Camino de los Descubrimientos s/n, 41092 Sevilla, Spain \\ e-mail:\{limon, alamo, eduardo\}@cartuja.us.es \\ †UFRGS - Depto. de Engenharia Elétrica \\ Av. Osvaldo Aranha 103, 90035-190 Porto Alegre-RS, Brazil \\ e-mail:jmgomes@eletro.ufrgs.br
}

Keywords: model predictive control, constrained control, control saturation, invariant sets, asymptotic stability.

\begin{abstract}
This paper is concerned with the design of stabilizing MPC controllers for constrained linear systems. This is achieved by obtaining a suitable terminal cost and terminal constraint using a saturating control law as local controller. The system controlled by the saturating control law is modeled by a polytopic differential inclusion. Based on this, it is shown how to determine a Lyapunov function and a polyhedral invariant set which can be used as terminal cost and constraint. The obtained invariant set is potentially larger than the maximal invariant set for the unsaturated linear controller, $\mathcal{O}_{\infty}$.

Furthermore, considering these elements, a dual-mode MPC is proposed. This controller guarantees the enlargement of the domain of attraction or, equivalently, the reduction of the prediction horizon for a given set of stabilizable initial states. If the local control law is the saturating LQR controller, then the proposed dual-mode MPC controller maintains the local optimality of the standard MPC. An illustrative example is given.
\end{abstract}

\section{Introduction}

MPC has become a popular control technique both in academy and industry. The main reason for this success is that MPC can be considered as an optimum control technique able to deal with constraints on the states and the manipulated variables in an explicit manner. Furthermore, a theoretical framework to analyze topics such as stability, robustness, optimality, etc. has been developed recently. See [9] for a survey, or [2] for process industry application issues.

It has been proved [9] that closed-loop stability of the MPC controller is guaranteed by adding a terminal cost and a terminal constraint in the optimization problem. The considered terminal cost is a Lyapunov function associated to the system controlled by a local control law. An associated invariant set is the terminal set. The domain of attraction of the MPC con- troller is the set of states that can be steered to the terminal region in $N$ steps, where $N$ is the prediction horizon.

The most common way of designing a stabilizing MPC controller for a constrained linear system is based on the following three ingredients: (i) an LQR as local stabilizing controller; (ii) a quadratic terminal cost equal to the optimal cost; (iii) a terminal set equal to the maximal admissible invariant set, $\mathcal{O}_{\infty}$. In this case, the obtained MPC controller is equal to the infinitehorizon optimal controller (constrained LQR) in a neighborhood of the origin.

If a saturating control law is used instead, then the region where the local controller is stabilizing can be increased. Therefore, the domain of attraction of the MPC controller can be enlarged (or, equivalently, the required prediction horizon can be reduced for a given set of stabilizable initial states). This idea has been previously used in [3] for single-input linear systems subject to constraint in the input (but not in the states). A region where the saturated LQR is optimal is presented and the optimal cost is explicitly computed for the closed-loop system. This cost is valid in the maximal invariant set contained in that region. This invariant set may be non-convex, which leads to a non-convex MPC optimization problem.

In this paper we use a saturating control law from a different point of view. From a differential inclusion representation, it is shown how to compute a suitable Lyapunov quadratic function and a polyhedral invariant set for the constrained system controlled by the saturating control law. The obtained invariant set is potentially larger than $\mathcal{O}_{\infty}$. Hence, the domain of attraction of the obtained stabilizing MPC controller may be enlarged. Furthermore, the obtained MPC is based on a convex quadratic programming problem and can be applied to generic constrained multi-input systems. In order to guarantee the enlargement of the domain of attraction remaining the local optimality of the LQR-based design, a dual MPC technique is proposed.

Notations. For any vector $x \in \mathbb{R}^{n}, x \succeq 0$ means that all the components of $x$, denoted $x_{(i)}$, are nonnegative and $x \succ 0$ means that are strictly positive. $y=|x|$, for $x \in \mathbb{R}^{n}$, denotes the component-wise absolute value, that is, $y_{(i)}=\left|x_{(i)}\right|$. For two vectors $x, y$ of $\mathbb{R}^{n}$, the notation $x \succeq y$ means that $x-y \succeq$ 0 . For a symmetric matrix $A, A>0$ means that it is definite 
positive. Consequently, for two symmetric matrices, $A$ and $B$, $A>B$ means that $A-B>0$. For a definite positive matrix $P>0, \varepsilon(P, \alpha)$ denotes the ellipsoid $\varepsilon(P, \alpha)=\left\{x \in \mathbb{R}^{n}\right.$ : $\left.x^{T} P x \leq \alpha\right\}$. $A_{(i)}$ denotes the $i$ th row of matrix $A$, and $A^{T}$ denotes the transpose of $A . I_{n}$ denotes the n-order identity matrix. For any vector $x \in \mathbb{R}^{n}$, $\operatorname{diag}(x)$ denotes the diagonal matrix obtained from $x$. Co $\{\cdot\}$ denotes a convex hull.

\section{Saturating Control Laws}

Let a discrete-time linear system be described by:

$$
x^{+}=A x+B u
$$

where $x \in \mathbb{R}^{n}$ is the current state of the system, $u \in \mathbb{R}^{m}$ is the current input and $x^{+}$is the successor state. The system is subject to hard constraints on state and control input:

$$
x(k) \in X, \quad u(k) \in U
$$

for all $k \geq 0$. The sets $X$ and $U$ are polyhedra containing the origin in their interior. Furthermore the set $U$ is given by

$$
U=\left\{u \in \mathbb{R}^{m}:|u| \preceq \rho\right\}
$$

where the vector $\rho \in \mathbb{R}^{m}$ is such that $\rho \succ 0$.

Consider that system (1) is stabilized by a state feedback law $u=K x$, that is, $K$ is such that the eigenvalues of $(A+B K)$ are placed inside the unit disk. Note that this control law is admissible inside a polyhedral region defined as:

$$
R_{L}=\left\{x \in \mathbb{R}^{n} ;|K x| \preceq \rho\right\}
$$

and hence, the control law $u=K x$ is only able to stabilize the constrained system in a subset of $R_{L} \cap X$. This set is the maximal positively invariant set for $x^{+}=(A+B K) x$ contained in $R_{L} \cap X$ (also called maximal admissible set) and denoted as $\mathcal{O}_{\infty}[4]$.

The controller might be extended outside this region, considering that the effective control law to be applied to the system is a saturated state feedback, where each component of the control vector is defined as follows:

$$
u_{(i)}=\left\{\begin{array}{cl}
-\rho_{(i)} & \text { if } K_{(i)} x<-\rho_{(i)} \\
K_{(i)} x & \text { if }-\rho_{i} \leq K_{(i)} x \leq \rho_{(i)} \\
\rho_{(i)} & \text { if } K_{(i)} x>\rho_{(i)}
\end{array}\right.
$$

For all $i=1, \cdots, m$. In this case, the closed-loop system becomes

$$
x^{+}=A x+B \operatorname{sat}(K x)
$$

which is a non-linear system.

Note that each component of the control law defined by (3) can also be written as $[6,5]$ :

$$
u_{(i)}=\operatorname{sat}\left(K_{(i)} x\right)=\alpha(x)_{(i)} K_{(i)} x
$$

where

$$
\alpha(x)_{(i)} \triangleq \begin{cases}\frac{-\rho_{(i)}}{K_{(i)} x} & \text { if } K_{(i)} x<-\rho_{(i)} \\ 1 \text { if } & -\rho_{(i)} \leq K_{(i)} x \leq \rho_{(i)} \\ \frac{\rho_{(i)}}{K_{(i)} x} & \text { if } K_{(i)} x>\rho_{(i)}\end{cases}
$$

with $0<\alpha(x)_{(i)} \leq 1, i=1, \ldots, m$.

The coefficient $\alpha(x)_{(i)}$ can be viewed as an indicator of the degree of saturation of the $i$ th entry of the control vector. In fact, smaller is $\alpha(x)_{(i)}$, farther is the state vector from the region $R_{L}$ given by Equation (2). Notice that $\alpha(x)_{(i)}$ is a function of the current state $x$. For the sake of simplicity, in the sequel we denote $\alpha(x)_{(i)}$ as $\alpha_{(i)}$. Hence, defining both $\alpha \in \mathbb{R}^{m}$ as a vector for which the ith entry is $\alpha_{(i)}, i=1, \ldots, m$, and a diagonal matrix $D(\alpha) \triangleq \operatorname{diag}(\alpha)$, the system (4) can be re-written as

$$
x^{+}=(A+B D(\alpha) K) x
$$

Note that the matrix $(A+B D(\alpha) K)$ depends on the current state $x$, since $\alpha$ does.

Consider now a vector $\underline{\alpha} \in \mathbb{R}^{m}$ such that $\underline{\alpha}_{(i)} \in(0,1]$ and define the following polyhedral region

$$
R_{L}(\underline{\alpha})=\left\{x \in \mathbb{R}^{n}:|K x| \preceq \rho(\underline{\alpha})\right\}
$$

where $\rho(\underline{\alpha})_{(i)}=\frac{\rho_{(i)}}{\underline{\alpha}_{(i)}}, i=1, \ldots, m$. Hence, for all $x \in$ $R_{L}(\underline{\alpha})$, it follows that $\underline{\alpha}_{(i)} \leq \alpha_{(i)} \leq 1, \forall i=1, \ldots, m$. Note that $R_{L} \subseteq R_{L}(\underline{\alpha})$.

From convexity arguments, for all $x$ belonging to $R_{L}(\underline{\alpha})$, it follows that: $D(\alpha) \in \mathbf{C o}\left\{D_{1}(\underline{\alpha}), D_{2}(\underline{\alpha}), \ldots, D_{2^{m}}(\underline{\alpha})\right\}$ where $\left\{D_{j}(\underline{\alpha})\right\}$ are diagonal matrices whose diagonal elements can assume the value 1 or $\underline{\alpha}_{(i)}$. Therefore, the system (4) can be locally represented by a polytopic model (or a polytopic differential inclusion) as it is stated in the following lemma [5, 6].

Lemma 1 Consider system (4) and a vector $\underline{\alpha} \in \mathbb{R}^{m}$ whose components $\underline{\alpha}_{(i)}, i=1, \ldots, m$ belong to the interval $(0,1]$. If $x \in R_{L}(\underline{\alpha})$, then successor state $x^{+}$derived from the system (4) can be computed by the following polytopic model:

$$
x^{+}=\sum_{j=1}^{2^{m}} \lambda_{j} A_{j}(\underline{\alpha}) x
$$

with $\sum_{j=1}^{2^{m}} \lambda_{j}=1, \lambda_{j} \geq 0$ and where

$$
A_{j}(\underline{\alpha}) \triangleq A+B D_{j}(\underline{\alpha}) K
$$

Note that the factors $\lambda_{j}$ may depend on the current state $x$. From this result the next lemma can be derived.

Lemma 2 If a set $\mathcal{S} \in \mathbb{R}^{n}$ contained in the region $R_{L}(\underline{\alpha})$ is positively invariant for the polytopic system (9), then $\mathcal{S}$ is positively invariant for the saturated system (4). 
Based on the polytopic differential inclusion representation (9), in the following lemma are given sufficient conditions to find a quadratic Lyapunov function for the saturated system (4). This lemma is particularly useful for the design of the stabilizing MPC controller proposed in section 5 .

Lemma 3 Consider system (4), a vector $\underline{\alpha} \in \mathbb{R}^{m}$ with $\underline{\alpha}_{(i)} \in$ $(0,1]$, and positive definite matrices $R \in \mathbb{R}^{m \times m}$ and $Q \in$ $\mathbb{R}^{n \times n}$. If there exists a positive definite matrix $P \in \mathbb{R}^{n \times n}$ satisfying the following LMI

$$
\begin{aligned}
& A_{j}(\underline{\alpha})^{T} P A_{j}(\underline{\alpha})-P \\
& +K^{T} D_{j}(\underline{\alpha})^{T} R D_{j}(\underline{\alpha}) K+Q<0
\end{aligned}
$$

for all $j=1, \ldots, 2^{m}$, then the function $F(x)=x^{T} P x$ verifies

$$
\begin{aligned}
F\left(x^{+}\right)-F(x) \leq & -x^{T} Q x \\
& -\operatorname{sat}(K x)^{T} \operatorname{Rsat}(K x)
\end{aligned}
$$

for all $x \in R_{L}(\underline{\alpha})$, where $x^{+}=A x+B \operatorname{sat}(K x)$.

Proof: It follows directly from the application of Schur's complement, convexity arguments and from representation of the saturated system given by Equation (9).

\section{Determination of the Terminal Set}

In this section, we show how to compute a suitable positively invariant set for system (4), based on the polytopic representation presented in the previous section. The procedure provides the maximal invariant set contained in $X_{L}=R_{L}(\underline{\alpha}) \cap X$ for the polytopic system (9).

Consider the following sequence of admissible sets for the system (9), given by

$$
\begin{aligned}
C_{0} & =X_{L} \\
C_{k} & =Q\left(C_{k-1}\right) \cap X_{L}, k \geq 1
\end{aligned}
$$

where the set $Q(\Omega)$ is the one-step set of $\Omega$, that is, the set of states that reach $\Omega$ in one step $[1,7]$.

The set $C_{k}$ is the region of initial states from which the system evolution remains in $X_{L}$ for the next $k$ sampling times. The sequence of admissible sets satisfies that $C_{k+1} \subseteq C_{k}$. The set $C_{\infty}$ is the set of states that are kept in $X_{L}$ for all the time, and hence is the maximum positively invariant set contained in $X_{L}$ for the polytopic system.

The one-step set to a polytope $\Omega=\left\{x \in \mathbb{R}^{n}: H x \preceq h\right\}$ for the polytopic system (9) is another polytope given by

$$
Q(\Omega)=\bigcap_{j=1}^{2^{m}} Q_{j}(\Omega)
$$

where $Q_{j}(\Omega)$ is the one-step set to $\Omega$ for the system $x^{+}=$ $A_{j}(\underline{\alpha}) x$, that is, $Q_{j}(\Omega)=\left\{x \in \mathbb{R}^{n}: H A_{j}(\underline{\alpha}) x \preceq h\right\}$.
Hence, provided that $X_{L}$ is a polytope, it follows that $C_{k}$ is a polytope, since it is the intersection of several polytopes.

In the next theorem it is stated that the maximum invariant set $C_{\infty}$ is finitely determined and it is a compact polytope, but first the following lemma is presented:

Lemma 4 If there exists a matrix of the polytopic model $A_{j}(\underline{\alpha})$ such that the pair $\left(K, A_{j}(\underline{\alpha})\right)$ is observable, then the set $C_{n-1}$ is a compact polytope, where $n$ is the order of the system.

\section{Proof:}

Let $C_{n-1}^{j}$ be the admissible set in $n-1$ steps for the system $x^{+}=A_{j}(\underline{\alpha}) x$ in the set $X_{L}(\underline{\alpha})$, i.e. $\mathcal{C}_{n-1}^{j}=\left\{x \in \mathbb{R}^{n}\right.$ : $\left.A_{j}(\underline{\alpha})^{i} x \in X_{L}, i=0, \cdots, n-1\right\}$. Then it is clear that $C_{n-1} \subseteq C_{n-1}^{j}$. Furthermore

$$
\mathcal{C}_{n-1}^{j} \subseteq\left\{x \in \mathbb{R}^{n}:\left|K A_{j}(\underline{\alpha})^{i} x\right| \preceq \rho(\underline{\alpha}), i=0, \cdots, n-1\right\}
$$

Taking into account that the observability matrix of $\left(K, A_{j}(\underline{\alpha})\right)$ is full-rank, then $C_{n-1}^{j}$ is compact and, hence, $C_{n-1}$ is compact.

Theorem 1 Let $V(x)=x^{T} P x$ be a Lyapunov function for the polytopic system (9) such that for all $x \in R_{L}(\underline{\alpha}), V\left(x^{+}\right) \leq$ $\mu V(x)$ where $\mu \in(0,1)$. If there exists a matrix $A_{j}(\underline{\alpha})$ such that $\left(K, A_{j}(\underline{\alpha})\right)$ is observable then:

(i) $C_{\infty}$ is finitely determined

(ii) $C_{\infty}$ is a positively invariant set for the saturated system (4), where it is exponentially stable and satisfies the constraints.

\section{Proof:}

From lemma 4 it is derived that the set $C_{n-1}$ is compact, and hence $C_{k}$ is compact for all $k \geq n-1$, since $C_{k} \subseteq C_{n-1}$.

Let $\varepsilon(P, \beta)$ denote the ellipsoid $\left\{x \in \mathbb{R}^{n}: x^{T} P x \leq \beta\right\}$. Since $C_{n-1}$ is bounded, there is a finite $\beta$ such that $C_{n-1} \subset$ $\varepsilon(P, \beta)$.

Let $\varepsilon(P, \gamma)$ be the maximum ellipsoid such that $\varepsilon(P, \gamma) \subset X_{L}$. Since $x^{T} P x$ is a Lyapunov function strictly decreasing for all $x \in X_{L}$, this set is a ( $\mu$-contractive )positively invariant set for the polytopic system and, hence, $\varepsilon(P, \gamma) \subset C_{\infty}$.

Note that $\varepsilon(P, \gamma) \subset C_{\infty} \subseteq C_{n-1} \subset \varepsilon(P, \beta)$ and hence $\beta \geq \gamma$. Let $M$ be a constant such that $\beta \mu^{M} \leq \gamma$, then, provided that $V\left(x^{+}\right) \leq \mu V(x)$, it follows that for all $x \in \varepsilon(P, \beta)$ the state of the system reaches $\varepsilon(P, \gamma)$ in $M$ steps or less.

Consider $i \geq n-1+M$, then $C_{i} \subseteq C_{n-1} \subset \varepsilon(P, \beta)$. Consequently, since $C_{i} \subseteq C_{M}$, for all $x \in C_{i}$ the system evolution is contained in $X_{L}$ and reaches $\varepsilon(P, \gamma) \subset C_{\infty}$ in $M$ steps or less. 
Therefore, for all $x \in C_{i}$, we have that the system remains in $X_{L}$ for all the time and hence $C_{i} \subseteq C_{\infty}$. This yields to $C_{\infty} \subseteq C_{i} \subseteq C_{\infty}$ which proves that $C_{i}=C_{\infty}$ and, therefore, it is finitely determined.

$C_{\infty}$ is the maximal invariant set contained in $X_{L}$ for the polytopic system (9) and then, since $X_{L} \subseteq R_{L}(\underline{\alpha})$, it is also a positively invariant for the saturated system (4). Furthermore, the existence of a strictly decreasing Lyapunov function $V(x)$ for the polytopic system, ensures the exponential stability of (4) in the set $C_{\infty}$.

Notice that the observability condition on $\left(K, A_{j}(\underline{\alpha})\right)$ is not necessary if the set $X_{L}$ is compact. This can be guaranteed if $X$ or $R_{L}(\underline{\alpha})$ are compact.

The obtained positively invariant set $C_{\infty}$ is a polytope, but it is not possible to ensure that the maximum invariant set for the unsaturated control law $\mathcal{O}_{\infty}$ is always contained in $C_{\infty}$. However, as could be seen in the numerical example, this inclusion often occurs or, at least, $C_{\infty}$ is potentially larger that $\mathcal{O}_{\infty}$.

Note that $C_{\infty}$ is contained in $R_{L}(\underline{\alpha})$ and in lemma $3, \underline{\alpha}$ is supposed to be given. Of course, in order to obtain a larger region $R_{L}(\underline{\alpha})$ and, as a consequence, a larger invariant set $C_{\infty}$, it is interesting to verify (10) with $\underline{\alpha}$ having components as small as possible. In the 1-input or 2-input cases, by applying a grid search, one can easily determine the minimal $\underline{\alpha}$ for which it is possible to find a solution for (10). Considering the generic multi-input systems, iterative schemes, as proposed in $[6,5]$, can be used.

For the computation of the admissible sets using the proposed procedure, an algorithm for removing redundant inequalities and another one for subset testing of polyhedra are necessary. There exists efficient algorithms for these tasks [7]. A different algorithm for the computation of $C_{\infty}$ based on linear programming schemes is given in [5].

\section{MPC Stability}

In the previous sections, a procedure to compute a Lyapunov function and an associated invariant set for the system controlled by a saturating control law has been proposed. These ingredients can be used to design a stabilizing MPC controller.

In MPC, the control action for a given state $x$ is obtained by solving an optimization problem $\mathcal{P}_{N}(x)$ defined by

$$
\begin{aligned}
V_{N}^{0}(x)= & \min _{\mathbf{u}} V_{N}(x, \mathbf{u}) \\
\text { s.t. } & \\
& u(j) \in U, x(j) \in X, j=0, \cdots, N-1 \\
& x(N) \in \mathcal{X}_{f}
\end{aligned}
$$

where $\mathbf{u}=\{u(0), u(1), \cdots, u(N-1)\}$ is a sequence of $N$ control actions, $V_{N}(x, \mathbf{u})$ is given by

$$
V_{N}(x, \mathbf{u})=\sum_{j=0}^{N-1} L(x(j), u(j))+F(x(N))
$$

where $L(x, u)=\|x\|_{Q}^{2}+\|u\|_{R}^{2}$, with $Q>0$ and $R>0$ and $x(j)=x^{\mathbf{u}}(j, x)$, that is the state at time $j$ if the initial state is $x$ at time 0 and the control sequence $\mathbf{u}$ is applied to the system.

At event $(k, x)$ problem $\mathcal{P}_{N}(x)$ is solved, yielding the minimizer $\mathbf{u}^{0}$ and the optimal cost $V_{N}^{0}(x)$. The MPC control law is implicitly given by $u=\kappa_{N}(x)=u^{0}(0)$.

In [9] the well-known sufficient conditions to guarantee asymptotic stability of the MPC controller are stated.

Theorem 2 [9] If the terminal set $\mathcal{X}_{f}$ is a positively invariant set for the system controlled by a local control law $u=\kappa_{f}(x)$ such that $\kappa_{f}(x) \in U$ for all $x \in \mathcal{X}_{f}$ and the terminal cost $F(x)$ is an associated Lyapunov function such that

$$
F\left(A x+B \kappa_{f}(x)\right)-F(x) \leq-L\left(x, \kappa_{f}(x)\right) \quad \forall x \in \mathcal{X}_{f}
$$

then $u=\kappa_{N}(x)$ asymptotically stabilizes the system for all feasible initial state, i.e. $x_{0} \in X_{N}\left(\mathcal{X}_{f}\right)$.

The domain of attraction $X_{N}\left(\mathcal{X}_{f}\right)$ may be enlarged by increasing the prediction horizon (which yields a greater computational burden) or increasing the size of the terminal set [8].

It is worth remarking that if one chooses the LQR as local controller $\kappa_{f}(x)=K_{L Q R} x$, the unconstrained optimal cost $F(x)=x^{T} P_{L Q R} x$ as terminal cost and the maximal invariant set $\mathcal{O}_{\infty}$ as terminal set, the obtained MPC control law is the infinite horizon optimal control law in a neighborhood of the origin.

\section{MPC controller design}

Based on the previous presented results, the following theorem can be stated.

Theorem 3 Consider a locally stabilizing controller $u=$ $\operatorname{sat}(K x)$ and let $P$ be the matrix solution of the equation (10) for given weighting matrices $Q$ and $R$ and for a given vector $\underline{\alpha}$. Suppose that $C_{\infty}$ defined from the sequence (12) is finitely determined. Then the MPC controller obtained by considering $F(x)=x^{T} P x$ as terminal cost and $\mathcal{X}_{f}=C_{\infty}$ as terminal set stabilizes asymptotically the system for all state in $X_{N}\left(C_{\infty}\right)$.

Note that this choice makes the domain of attraction of the MPC potentially larger that the one based on the unconstrained local controller and the resultant optimization problem is a convex quadratic programming one.

Although a saturated LQR, $u=\operatorname{sat}\left(K_{L Q R} x\right)$, is used as local controller, the designed MPC may not be the infinite-horizon 
optimal in a neighborhood of the origin. This is due to the fact that the considered terminal cost is a conservative approach to the optimal cost of the controller and hence, $P>P_{L Q R}$. In order to reduce this conservativeness, the matrix $P$ is computed by solving (10) minimizing its trace. Furthermore, the obtained terminal set $C_{\infty}$ may not include $\mathcal{O}_{\infty}$.

These drawbacks can be overcome by a simple procedure to implement the controller:

- If $x \in X_{N}\left(\mathcal{O}_{\infty}\right)$, then consider $F(x)=x^{T} P_{L Q R} x$ and $\mathcal{X}_{f}=\mathcal{O}_{\infty}$.

- Else, consider $F(x)=x^{T} P x$ and $\mathcal{X}_{f}=C_{\infty}$.

The control law is obtained by solving the resultant optimization problem which yields a dual-mode control law. This controller asymptotically stabilizes the system in a domain of attraction $X_{N}\left(C_{\infty}\right) \cup X_{N}\left(\mathcal{O}_{\infty}\right)$ and hence, the enlargement property is ensured. Moreover, the infinite horizon optimality property of the MPC holds.

The condition $x \in X_{N}\left(\mathcal{O}_{\infty}\right)$ can be easily checked, since this set is a polyhedral that can be computed efficiently off-line [7]. Another technique is checking the feasibility of the associated optimization problem for a given $x$, which can be posed as an LP problem.

As it was mentioned before, the MPC design based on a saturated LQR proposed in [3] maintains the optimality property of the MPC at expense of using a non-convex terminal set (and hence a non-convex optimization problem). This nonconvexity can be overcome by choosing a large enough prediction horizon. This procedure increases the computational burden and may reduce the enlargement of the domain of attraction derived from the proposed design. Furthermore, this technique is only valid for single-input systems.

The design presented in this paper provides a polytope and a quadratic terminal cost for multiple-input systems subject to constraints on states. This yields a stabilizing MPC derived from a convex optimization problem. Furthermore, by using a simple dual-mode MPC controller, the optimality and the enlargement properties are guaranteed.

\section{Numerical Example}

Consider a system $x^{+}=A x+B u$ given by

$$
A=\left[\begin{array}{ll}
1 & 1 \\
0 & 1
\end{array}\right] \quad B=\left[\begin{array}{ll}
0 & 0.5 \\
1 & 0.5
\end{array}\right]
$$

where the inputs are constrained to $\|u\|_{\infty} \leq 0.3$ and $\|x\|_{\infty} \leq$ 2. For this system, a LQR controller with $\bar{Q}=I_{2}$ and $R=\bar{I}_{2}$ is computed. The controller $u=K x$ and the optimal cost $F(x)=x^{T} P_{L Q R} x$ are given by

$$
K=\left[\begin{array}{ll}
-0.0037 & -0.5850 \\
-0.5919 & -0.8844
\end{array}\right] \quad P_{L Q R}=\left[\begin{array}{ll}
2.1801 & 1.1838 \\
1.1838 & 2.7688
\end{array}\right]
$$

The maximal invariant set for this controller, $\mathcal{O}_{\infty}$, is shown in figure 1.

Following the technique proposed in the paper, a Lyapunov function and a positively invariant set for the saturating control law derived from the LQR controller is computed. First, it has been chosen a vector $\underline{\alpha}$ such that the LMI (10) is feasible. The obtained vector is $\underline{\alpha}=[0.25,0.2]$ and the calculated quadratic terminal cost $F(x)=x^{T} P x$ is given by

$$
P=\left[\begin{array}{cc}
33.5508 & 28.2391 \\
28.2391 & 208.3942
\end{array}\right]
$$

Next, the maximal invariant set, $C_{\infty}$, for the polytopic system contained in $X_{L}=R_{L}(\underline{\alpha}) \cap X$ is calculated. Both sets are depicted in figure 1 . Note that $\mathcal{O}_{\infty} \subset C_{\infty}$.

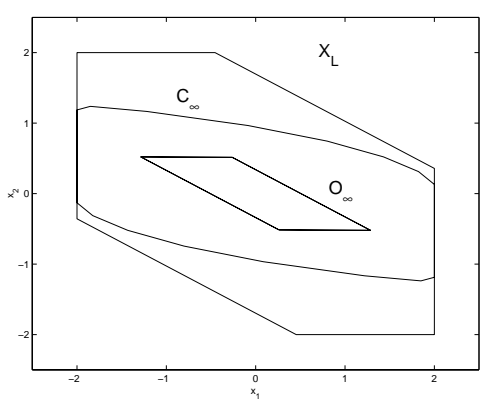

Figure 1: Terminal sets $\mathcal{O}_{\infty}$ and $C_{\infty}$.

Considering the terminal cost and terminal set obtained, a stabilizing MPC can be computed. The domain of attraction of the MPC based on the unsaturated LQR controller, $X_{N}\left(\mathcal{O}_{\infty}\right)$, is contained in the one of the MPC based on the saturating control law, $X_{N}\left(C_{\infty}\right)$. This enlargement is shown in figure 2 for an MPC with $N=2$.

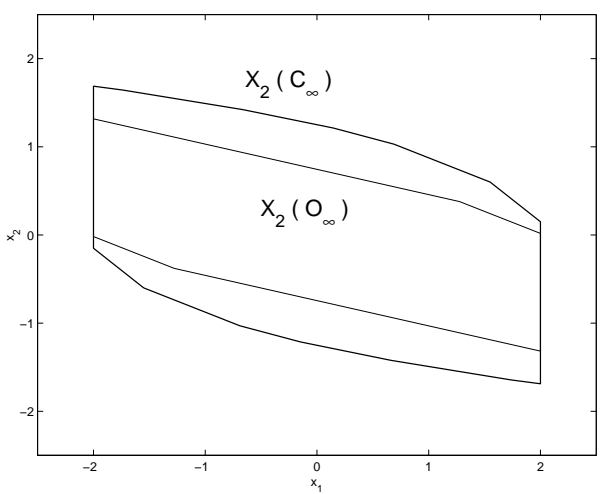

Figure 2: Domain of attraction of the MPC controller with each terminal set.

Note that the enlargement of the domain of attraction is equivalent to a reduction of the prediction horizon. In figure 3 , it is shown that $X_{4}\left(\mathcal{O}_{\infty}\right) \subset X_{2}\left(C_{\infty}\right)$. Therefore, all state stabilizable by the LQR based MPC with $N=4$ is stabilizable by the MPC based on the saturated LQR with $N=2$. Furthermore, in this case, $X_{4}\left(C_{\infty}\right)$ is equal to the maximal stabilizable set $X_{\infty}$. Hence, for $N=4$, the MPC based on the saturating con- 


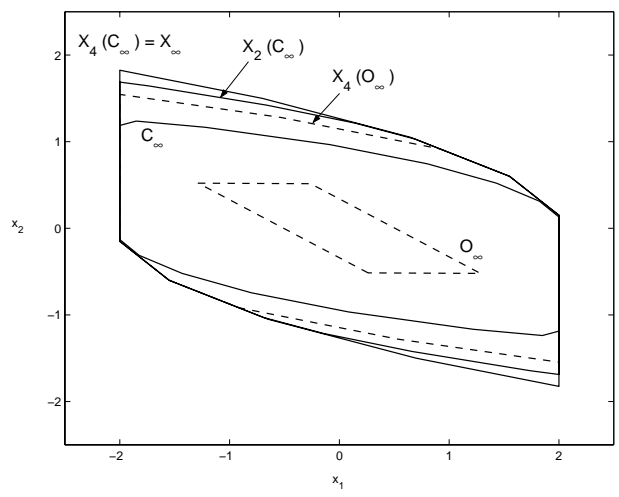

Figure 3: Comparison between domains of attraction of the MPC for several prediction horizons.

trol law is able to stabilize all stabilizable set, while the MPC based on the LQR is not.

However, although the LQR based MPC is the optimal controller, the MPC based on the saturating LQR is not. In order to improve the optimality of the controller, a dual MPC is proposed. In figure 4 , it is compared the evolution between the dual MPC and the standard MPC based on the saturating control law for four initial states. In Table 1 the cost associated to the evolution of the closed loop system for both controllers is compared. It is demonstrated that the dual-mode MPC controller presents a lower cost, and hence, the performance of the closed loop system is better.

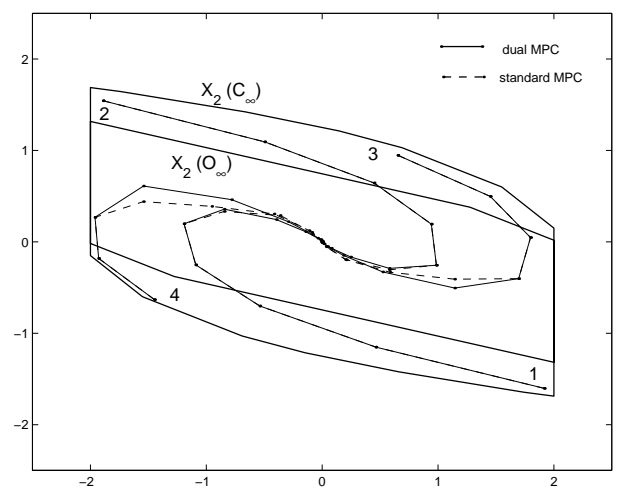

Figure 4: Closed loop state portrait of the dual MPC and the standard MPC.

\begin{tabular}{c|cc}
$x_{0}$ & dual & standard \\
\hline 1 & 13.2857 & 13.3125 \\
2 & 11.3655 & 11.3759 \\
3 & 12.7831 & 12.8275 \\
4 & 14.6420 & 14.8448
\end{tabular}

Table 1: Comparative of the cost of the evolution of standard MPC and the proposed dual-mode MPC

\section{Conclusions}

In this paper we present a technique to design a stabilizing MPC controller for constrained linear systems, which is based on a saturating control law. Using a polyhedral differential inclusion for representing the behaviour of the closed-loop system, a quadratic terminal cost and a polytopic invariant set can be efficiently computed. This set is potentially larger than the maximal invariant set for the system controlled by the unsaturated controller. These ones can be used to design a stabilizing MPC controller with an associated convex optimization problem. Furthermore, a dual-mode MPC controller is presented. This approach guarantees the enlargement of the domain of attraction and keeps the local optimality property derived from using a LQR as local controller.

\section{References}

[1] F. Blanchini. Ultimate boundedness control for discretetime uncertain system via set-induced lyapunov functions. IEEE Transactions on Automatic Control, 39:428-433, 1994.

[2] E. F. Camacho and C. Bordons. Model Predictive Control. Springer-Verlag, 2 edition, 1999.

[3] J. A. De Doná, M. M. Seron, D. Q. Mayne, and G. C. Goodwin. Enlarged terminal sets guaranteeing stability of receding horizon control. Systems \& Control Letters, 47:57-63, 2002.

[4] E. G. Gilbert and K. Tan. Linear systems with state and control constraints: The theory and application of maximal output admissible sets. IEEE Transactions on Automatic Control, 36:1008-1020, 1991.

[5] J. M. Gomes Da Silva Jr. and S. Tarbouriech. Stability regions for linear systems with saturating controls. In Proceedings of the ECC, 1999.

[6] J. M. Gomes Da Silva Jr. and S. Tarbouriech. Local stabilization of discrete-time linear systems with saturating controls: an LMI-based approach. IEEE Transactions on Automatic Control, 46:119-125, 2001.

[7] E. C. Kerrigan. Robust Constraint Satisfaction: Invariant Sets and Predictive Control. PhD thesis, University of Cambridge, 2000.

[8] D. Limon, T. Alamo, and E. F. Camacho. Enlarging the domain of attraction of MPC controller using invariant sets. In Proceedings of the IFAC World Congress, 2002.

[9] D. Q. Mayne, J. B. Rawlings, C. V. Rao, and P. O. M. Scokaert. Constrained model predictive control: Stability and optimality. Automatica, 36:789-814, 2000. 